\title{
THE RECENT TREND OF RADIO DIRECTION-FINDING
}

$\mathrm{T}^{\mathrm{H}}$ HE radio direction-finder is an instrument for determining the direction of arrival of electric waves of the frequencies normally used in radio communication. Although it was introduced during the early years of the present century, it did not become a practically useful instrument until the valve-amplifying receiver was developed and provided the necessary sensitivity in the reception of radio signals. In its early form, the radio direction-finder, using closed loop aerials and operating on wave-lengths of from a few hundred to several thousand metres, was applied as an aid to marine navigation, and as a means of locating the position of unknown transmitting stations ; in addition, however, the instrument provided a very useful tool in connexion with the study of the mode of propagation of electric waves.

Arising out of these applications, it was noticed that under certain conditions of wave propagation and beyond certain minimum distances of transmission, the directional indications of the instruments were subject to large and variable errors, which were found to be due to the action of the horizontal components of electric force in the arriving waves on the horizontal sides of the receiving loop. The remedy for this state of affairs was to be found in a British patent applied for by $F$. Adcock in 1918, in which it was proposed to replace the closed receiving loops by. pairs of spaced vertical aerials, so connected together that the resultant action of any horizontal components of electric force could be reduced to a negligible amount. The practical utility of this invention was demonstrated in 1926, and soon afterwards the Adcock direction-finder became a commercial proposition for medium wave-lengths.

This advance had the effect of increasing the range of usefulness of ground receiving stations to several hundred miles, at which distances polarization errors ranging up to $45^{\circ}$ or more with the loop aerial were reduced to less than one tenth of this amount with the spaced vertical aerial. Limitations of space have delayed the application of this improved direction-finding system on board ship, but the closed loop type used on medium wave-lengths has so far been found sufficiently accurate for the comparatively modest range requirements of marine navigation. Since about 1930, two factors have had a major effect upon the trend of radio direction-finding investigations and their application. In the first place, the growth of aerial transport has given rise to a demand for accurate radio bearings of aircraft at distances ranging up to several thousand instead of several hundred miles ; and secondly, the general trend of radio communica. tion to shorter and shorter wave-lengths has necessitated the extension of direction-finding technique in a corresponding manner. For the particular case of aerial navigation, various radio beacons have been developed by which a pilot may be guided to an acrodrome and enabled to land under conditions of poor visibility, but the scope of such aids is usually limited to comparatively short ranges, except in the case of long straight air routes, such as exist in the United States of America.

In order to provide a useful radio-bearing service to aircraft at ranges between 200 and 2,000 miles, it is necessary to resort to the use of short waves, so that the comparatively minute transmitter power available on the aircraft may produce sufficient field intensity at the ground receiving station. For this purpose, spaced aerial directionfinders have been developed to a high standard of performance for the wave-length band 10-100 metres. Such instruments are now available, with either a simple aerial receiver in which the bearing is determined by rotating a goniometer search coil to a zero or minimum signal position, or a dual receiver and cathode-ray tube indicator in which the bearing is read from a visual trace on the fluorescent screen.

A detailed theoretical and experimental study of the principles of design and construction of such short-wave direction-finders has led to the conclusion that their inherent instrumental accuracy should be of the order of $1^{\circ}$, with maximum error values of $2^{\circ}$ or $3^{\circ}$ under the most severe conditions of reception of horizontally polarized waves. When such direction-finders were used in practice, however, it was found that the observed bearings of transmitting stations in known positions were subject to errors much greater than this, sometimes ranging up to $10^{\circ}$ or $20^{\circ}$. This raised the question as to whether the instrument was still defective due to some undetected fault, or whether the measurements indicated departures of the arriving waves from the great circle plane between transmitter and receiver.

To assist in the resolution of this question, resort was had to an alternative means of direction finding, which, although first described by T. L. Eckersley in 1921, has not been brought into use as a practical instrument until within the past few years. In this case, the reception takes place on a pair of identical vertical closed loops, spaced a 
fraction of a wave-length apart and symmetrically connected to the central receiver in such a manner that certain instrumental defects inherent in the use of open aerials can be eliminated. It is convenient to use a visual receirer of the cathode-ray tube type, and various tests have demonstrated that the inherent instrumental accuracy of such apparatus is somewhat higher than that previously attainable.

When used for taking bearings on short-wave transmitting stations, however, it was found that the accuracy was not materially better than that hitherto obtained. It is to be concluded, therefore, that on the short wave-lengths under consideration, a severe limit is set to the accuracy of radio direction finding under certain conditions by vagaries in the propagation of the electric waves employed. The direction-finding instruments indicate the actual direction of arrival of the waves to a high order of accuracy, but this is not necessarily the direction of the transmitter, but rather that of the point of reflection or scattering of the waves from the ionosphere. It is perhaps fortunate that it is possible to avoid using the bearings obtained under such conditions, and increased knowledge and experience will soon indicate in precisely what circumstances it is safe to rely upon the radio bearings obtained. Meanwhile, the use of these short-wave direction. finders is adding considerably to our knowledge of the mode of propagation of electric waves through the ionosphere.

In the range of wave-lengths below 10 metres, direction finding is in the early stages of development, but instruments of the closed loop and spaced aerial types have already been used for ascertaining the possibilities of the art on wavelengths down to 3 metres. It seems likely that within this wave-length band, some application is likely to be found in ascertaining the positions of radio sounding balloon transmitters at ranges up to one hundred miles or so. In this sphere, however, much more research is needed on the propagation of waves as well as on the design and construction of the direction-finding instruments.

R. L. S.R.

\title{
BIOLOGY, THE SCHOOL AND SOCIETY*
}

\author{
By T. H. HAWKINS \\ Education Officer, British Social Hygiene Council
}

$\mathrm{T}^{\mathrm{H}}$ HE acceleration of scientific invention during the last generation has been such that it has now considerably outdistanced the ability of man to assimilate knowledge and to make it an essential part of his social and ethical relationships. This was the theme of the address given before the Ninth Imperial Social Hygiene Congress by Prof. J. Ritchie, of the University of Edinburgh, who suggested that the failure is in some measure due to the exclusiveness of science, and that its remedy partly lies in the creation of a world-wide appreciation of the social and moral implications of scientific knowledge. That this appreciation can in part be brought about by biological knowledge was the keynote of the educational sessions of the Congress, which was attended by delegates from all parts of the Empire.

At the opening session, the speakers concerned themselves with the teaching of biology as a factor in health and character training. Mr. C. R. E. Gillett, University. of Reading, in comparing the older public and newer State schools, suggested that the latter are now attempting to turn out a product which hitherto has presumably belonged

- Survey of the educational sessions of the Ninth Imperial Social Hygiene Congress, held in the Hastings Hall, British Jedical Association House, July 10-14. exclusively to the former, namely, a citizen qualified to play his part in our changing form of democratic government. This citizen would be keenly aware of his responsibilities to the community and would be imbued with such a sense of social duty that he would readily undertake those tasks which contribute to the welfare and establishment of communal life. But whether the will to serve has demonstrably expressed itself is a matter of great doubt, and here only education would make it effective. The part that biology can play in health and character formation will only become apparent if the pupil be made to feel keenly aware of the value of his training. Active co-operation will be needed between teachers of physical training, domestic science, and biology, and this should be unified by a 'positive' attitude towards health on the part of the school staff as a whole. The part of the biological teacher in this co-ordinated form of health education would be that of the true scientific investigator, seeking with the children to elucidate real problems, rather than being a mere imparter of factual information. If children were allowed to cooperate fully in the elucidation of simple medicobiological problems, they would not fail to develop 